\title{
The Standard, Power, and Color Model of Instrument Combination in Romantic-Era Symphonic Works
}

\author{
RANDOLPH JOHNSON \\ School of Music, The Ohio State University
}

\begin{abstract}
The Standard, Power, and Color (SPC) model describes the nexus between musical instrument combination patterns and expressive goals in music. Instruments within each SPC group tend to attract each other and work as a functional unit to create orchestral gestures. Standard instruments establish a timbral groundwork; Power instruments create contrast through loud dynamic climaxes; and Color instruments catch listeners' attention by means of their sparing use. Examples within these three groups include violin (Standard), piccolo (Power), and harp (Color). The SPC theory emerges from analyses of nineteenth-century symphonic works. Multidimensional scaling analysis of instrument combination frequencies maps instrument relationships; hierarchical clustering analysis indicates three SPC groups within the map. The SPC characterization is found to be moderately robust through the results of hypothesis testing: (1) Color instruments are included less often in symphonic works; (2) when Color instruments are included, they perform less often than the average instrument; and (3) Color and non-Color instruments have equal numbers of solo occurrences. Additionally, (4) Power instruments are positively associated with louder dynamic levels; and (5) when Power instruments are present in the musical texture, the pitch range spanned by the entire orchestra does not become more extreme.
\end{abstract}

Submitted 2009 August 11; accepted 2011 March 13

KEYWORDS: orchestration theory, instrument combination, musical gesture

MUTUAL instrument-family membership implies that certain instruments have a similar timbre, but studies suggest that shared family does not always correlate with common, perceived timbre. John Grey (1977) investigated listeners' perception of timbre similarities between instruments: he observed that acoustical properties of instruments can "override the tendency for instruments to cluster by family" ( $p$. 1276). The questions and methods of Grey's study extend through several decades of timbre research (e.g., Caclin, McAdams, Smith, \& Winsberg, 2005; McAdams, Winsberg, Donnadieu, De Soete, \& Krimphoff, 1995). These studies explore the perceptual dimensions of timbre: spectral centroid and attack time are well-established timbral elements. The remaining dimensions are generally acknowledged to be types of spectral or spectral-temporal cues (Caclin et al., 2005). Despite the importance of these findings, they have yet to be fully applied to music analysis and the study of orchestration. A timbre-based perspective on instrument similarity and dissimilarity has important applications to understanding orchestration.

For example, Grey's study demonstrated that mutes drastically alter tone color: the oboe and muted trombone were heard as similar. Additionally, instruments can have fluctuating timbre characteristics depending on register: a high-range bassoon tone was judged as similar to a brass tone. These observations suggest that acoustic parameters might influence the instrument groupings used in real musical situations, where instruments change roles flexibly according to their manner of performance.

In contrast to the timbre model, the instrument-family model classifies instruments according to their physical attributes and mode of sound production (e.g., bowed or struck); this is an important taxonomy, but families do not automatically translate into the ideal groups to be used in a composition. Despite orchestration manuals' pedagogical soundness with regard to instrumentation topics (e.g., range, dynamics, fingerings), the some chapter titles can have a hindering implication for orchestration: that the family membership of instruments largely determines combination choices to be made in orchestration.[1] This could discourage the use of combinations between instrument families. The present study hopes to 
encourage expressive orchestration through an investigation of this aspect of orchestration theoryinstrument combination patterns.

Both family and timbre comparisons commonly explain instrument-combination choices in orchestration, but which interpretation more precisely describes instrument combination patterns? Are there other accounts of instrument combination patterns that reveal the fundamental, expressive principles behind orchestration?

The answer might come in a form that fuses traditional orchestration studies with recent psychological research. Walter Piston expressed his desire to merge scientific inquiry with the art of orchestration when he discussed the advantages of organizing the musical variables involved in orchestration (Piston, 1955, p. viii). Traditionally, orchestration has been learned through extensive score study and hands-on experience; new teaching and study techniques should still embrace these valuable endeavors, but also enhance them with applications gleaned from timbre research.

Along these lines, the current study proposes another explanation of instrument combination: grouping according to the goals of orchestral gestures. This gestural model explores the basic groups of instruments that composers choose to deploy during their works. Supposing that a composer wanted to use a homogenous tone-color mixture: she would then combine instruments with similar timbre. This goal of "blend," see Sandell (1991), is common (especially in Romantic-era compositions) and timbre similarity likely influences instrument combination choices. In an alternative example, timbre similarity might not correlate with instrument combination choices: sometimes composers create an impression of a multi-part dialogue between "characters" in the orchestra. In this case, contrasting instruments are obvious combination choices because dissimilarity promotes voice independence.

Instrument-family classification is a commonly used instrument categorization, but it does not fully describe the phenomenon of instrument combination. Timbre theory makes a major step forward through its consideration of instruments' perceptual similarities, which parallel acoustic attributes (Caclin et al., 2005). The present study combines timbre theory with a theory of orchestral gestures. For the purposes of this study, orchestral gestures are defined as devices that composers use to repeat, vary, and connect phrases. Gestures range in length from several measures to a whole section of a piece. The "Rossini crescendo" and Stravinsky's sudden changes of block textures are examples of orchestral gestures. Gestures not only function as articulators of musical form, but they also carry qualities such as a "smooth build" (Rossini) and "sudden interruption" (Stravinsky). A model of orchestration that combines theories of timbre and gesture has the potential to richly characterize the instrument combination patterns in symphonic works.

In brief, the current study constructs a new model of instrument combination based on patterns of instrument use in a corpus of nineteenth-century symphonies. Several exploratory statistical techniques show actual instrument groupings without filtering the results through an a priori model of orchestration. The results are incorporated into a new model called the Standard, Power, Color (SPC) model of instrument combination. In the last stage of the study, the SPC model is used to produce a number of hypotheses related to orchestration. The hypotheses are then tested using new samples from nineteenth-century symphonies.

\section{EXPLORATORY MODEL}

Research questions and methods from timbre studies sparked phase one of the present study. Multidimensional scaling models have been used for several decades to visualize timbre space. The current study used a similar method, but it was applied to new data: the combination frequencies of instruments. The model proposed here is the Standard, Power, and Color model (hereafter, the SPC model). The SPC model is driven by an interest in function-with emphasis on the questions of "why" and "how" instruments combine and lead to musical gestures.

The population encompassed by the scope of the present study consisted of Romantic-era symphonic works for orchestra. Pieces that feature a solo instrument (e.g., concertos) or voices were excluded. It was impractical to study every work in the Romantic tradition; therefore, a representative sample sufficed. The idea of a musical "canon" has been hotly contested in musicological discussions over the past two decades. Without endorsing some concept of musical "greatness," it nevertheless was useful to sample a representative group of works from the commonly accepted central orchestral repertoire. The present study operationally defined "nineteenth-century symphonic works" as the compositions listed in "Part IV - The Romantic Age" of David Dubal's The Essential Canon of Classical Music (2001). Included 
pieces were those listed as "orchestral works" or those identified as a "symphonic poem" in their titles or descriptions. Two hundred thirty pieces $(n=230)$ met these criteria.

Since orchestration techniques in the twentieth century became highly diversified, exploratory, and strongly tied to individual composers, the present treatment of orchestration is limited to the nineteenth century in the interest of studying a manageable number of variables. This time period witnessed fruitful advances in instrument construction technology that led to an expanding palette of instrument colors. The Romantic-period symphony is often considered the culmination of the Classical symphony and the bridge to the Modern symphony.

\section{Method}

Fifty orchestral sonorities (harmonic snapshots in time) made up a chord database: a single sonority was sampled randomly from each of 50 randomly-selected symphonic works from the canon. (50 works were sampled from a total of 230 works so that later hypothesis tests could draw on a reserve data set.) A random number generator was used to determine the piece selected from the canon; the page number within each piece; and the page location (ruler measurement) of each sonority. (New random page and ruler measurement numbers were used for each piece.) Each entry in the chord database contained the names of instruments that sustained or articulated pitches at the sampled moment.

After the sample was completed, custom-programmed computer scripts helped to sort through all of the possible instrument-pair combinations and calculate the frequency that each instrument pair performed together; frequencies ranged from 0.0 (the two instruments never played at the same time) to 1.0 (the two instruments always performed in tandem). These frequencies were then subtracted from 1: this transformed them into abstract "distance" values between instruments. Greater distance between two instruments represented their infrequent combination; smaller distance represented more frequent combination. Percussion instruments (other than the timpani) were not included in this model due to their insufficient representation in the sample.

\section{Results}

A multidimensional scaling (MDS) analysis used the abstract distance values between instruments as dissimilarity measures.[2] In the current study, stress is a measure of how well the MDS model organizes the observed distances between instrument pairs. Although adding more and more dimensions can continually reduce the stress on a MDS model, there is a point of diminishing returns. Statisticians recommend that the number of dimensions just before the point of diminishing returns should be adopted as the best balance between lower stress and easier interpretability. For the current data set, the ideal balance between low stress and fewer dimensions occurred with the three-dimensional (3D) solution. Although the 3D solution was ideal, it is possible to display one of the most interpretable angles of the 3D solution in a two-dimensional (2D) depiction. Table 1 summarizes the instruments examined, gives their abbreviations, and indicates their membership in the categories of the SPC model. For visual clarity, Figure 1 collapses the $3 \mathrm{D}$ solution into a $2 \mathrm{D}$ solution. 


\begin{tabular}{|l|l|l|}
\multicolumn{1}{l|}{ Classification } & \multicolumn{1}{l}{ Instrument } & \multicolumn{1}{l|}{ Abbreviation } \\
\hline Standard & Clarinet & clar \\
Instruments & Flute & flt \\
& Bassoon & fagot \\
& Oboe & oboe \\
& Viola & viola \\
& Violin & violn \\
& String bass & cbass \\
& Violoncello & cello \\
\hline Power & Horn & cor \\
Instruments & Trumpet & tromp \\
& Timpani & timpa \\
& Trombone & tromb \\
& Tuba & tuba \\
& Piccolo & picco \\
\hline Color & Bass clarinet & bclar \\
Instruments & English horn & cangl \\
& Harp & arpa \\
& Cornet & cornt \\
& Contrabasson & fag_c \\
\hline
\end{tabular}

Table 1. Instruments investigated, abbreviations, and classification in the Standard, Power, and Color model.

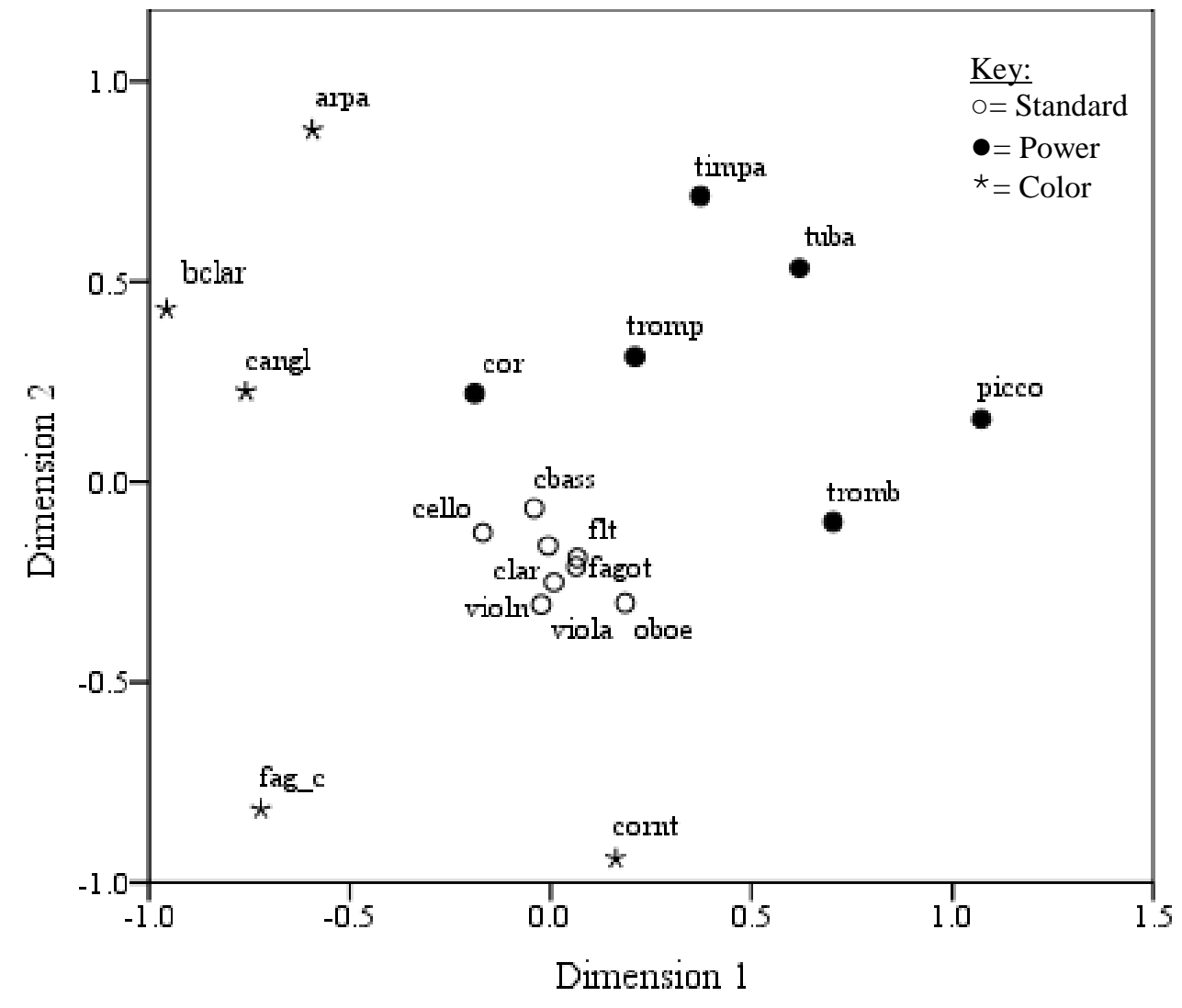

Figure 1. Multidimensional scaling map of instruments' proximity according to frequency of combination; dimensions are abstract and interpretable in different ways. 
The MDS model's dimensions are not specified automatically by the statistical calculationdimensional interpretation in this case came from reconciling the abstract map with the researcher's music experience.

In the map's center is a nucleus containing very similar (i.e., frequently combined) instruments: violoncello, string bass, viola, clarinet, violin, bassoon, flute, and oboe. (The horn is a bit further away from the center, but could conceivably be a part of the nucleus.) In terms of the instrument family model, this nucleus is the string section plus the core woodwind instruments. The present study's new instrument combination terminology will refer to this central group of instruments as "Standard" instruments.

Their qualities are listed below:

\section{STANDARD INSTRUMENTS}

- Perform for the majority of the time

- Cover a broad pitch range from the lows of the string bass to the highs of the violin and upper woodwinds

- Assume roles flexibly in both melody and accompaniment

- Are dynamically moderate

Another group is located on the upper right side of Figure 1; this group contains the brass section, timpani, and, surprisingly, the piccolo. These instruments are not as closely spaced as the Standard instruments, but they occupy a region distinct from the rest of the orchestra. This is suggested by the gap on the map. The word, "Power," best encapsulates the traits of this group:

\section{POWER INSTRUMENTS}

- Are dynamically intense in their idiomatic usage

- Cover the middle and extremes of the pitch spectrum without sacrificing loud dynamic levels

The third group emerging from the map is the most diffuse of all in terms of the instruments' proximity to each other, but they all share the extreme-left periphery on the map. These instruments are deemed "Color" instruments:

\section{COLOR INSTRUMENTS}

- Perform more softly than other instruments

- Are modified versions (different bore type or instrument length) of more common instruments

- Are used less commonly

- Work well as unique soloists (especially the harp and English horn)

Dimension 1 on the map can be interpreted as the dynamic potential of the instruments: characteristically louder instruments are at the right side of the map. Dimension 2 is more difficult to interpret, and is perhaps a product of the particular rotation of the MDS output. The groups of instruments and the distances between groups are more important than assigning a label to dimension 2.

To assist further with the analysis, a hierarchical clustering analysis (Figure 2) bolstered the interpretation of groups within the MDS model.

The clustering analysis (divisive method) began with all instruments in one group and then gradually split the group into smaller groups until each instrument became its own category. The ideal balance between fewer numbers of groups and the stress on the model occurred at case 12, where the stress experienced a large reduction followed by minimal reductions at smaller case numbers. After this point, further splits into smaller categories did not account for very large differences between instruments. The vertical line at case 12 intersects three branches of the dendrogram; this suggests three instrument groups in the clustering analysis and MDS map. 


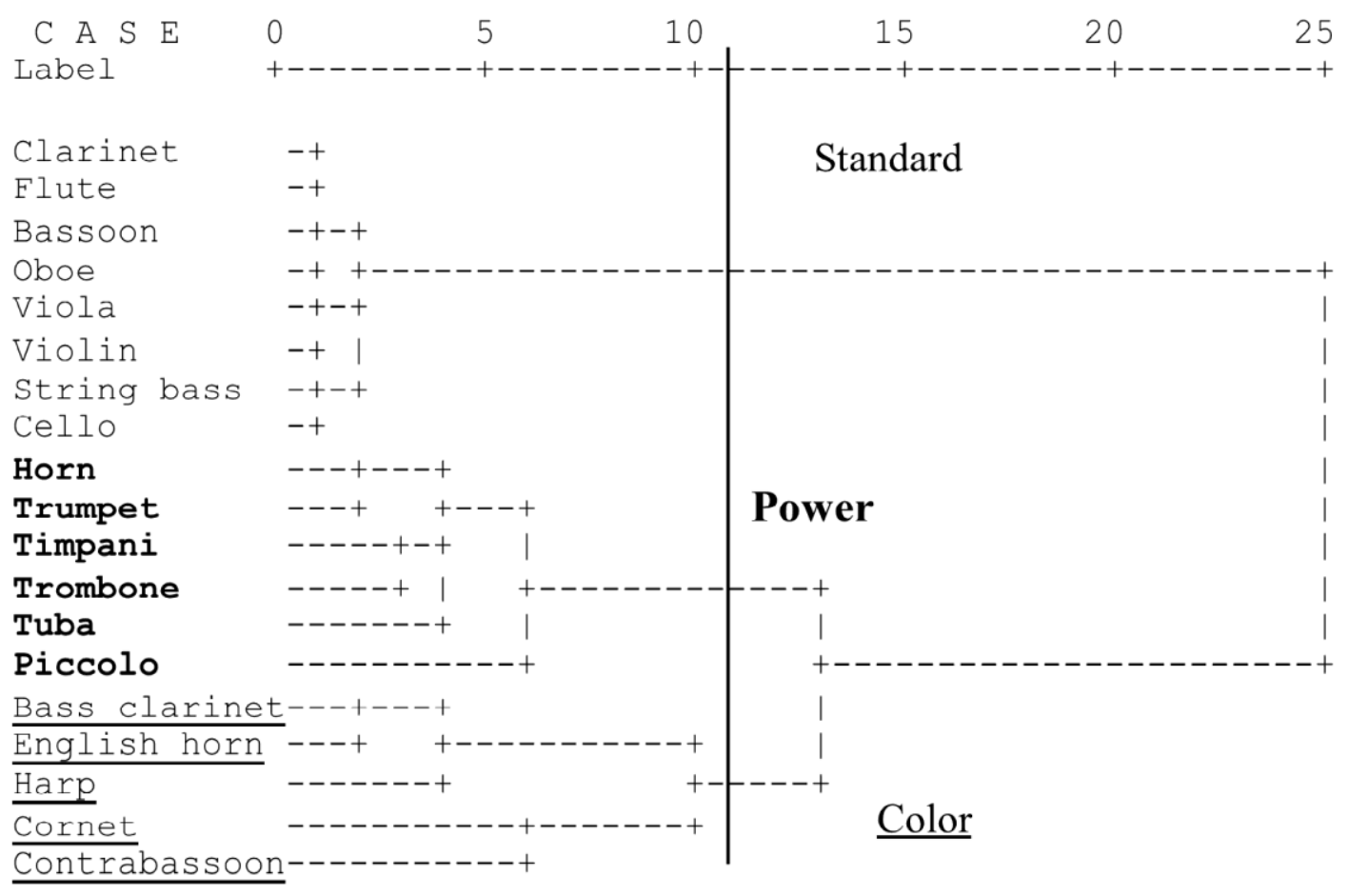

Figure 2. Dendrogram of hierarchical clustering analysis; the three-group solution can be observed at case number "12."

The MDS map and hierarchical clustering analysis highlighted three instrument deployment groups in a new model of instrument combination in orchestration - the Standard, Power, and Color model. This classification scheme emphasizes the function of instruments rather than their physical form or common manner of acoustic activation.

\section{Conclusion - The SPC Model}

Instrument combination frequency is an important consideration in orchestration. At the simplest level, an orchestrator might want quick solutions in the form of heuristics that address the questions, "What instruments will sound like they belong together?" or "What other instrument can I combine with this instrument to produce an odd or uncommon tone mixture?" More deeply, combination frequencies might indicate underlying gestures that are important in orchestration.

Standard instruments were so-named because they form the nucleus of the orchestra through their frequent inclusion in symphonic works. Power instruments all have an ability to perform at high amplitudes; thus, it is predicted that they are used especially to support loud dynamic levels. The Power group includes some instruments that occupy pitch extremes (e.g., piccolo and tuba), so it is predicted that the group might also have associations with the overall pitch range of the orchestra. One important attribute of Color instruments would be their relatively rare use in symphonic works; if they were held in reserve, then the moments when they enter would bring fresh and unexpected tone colors. Consequently, one could predict that Color instruments are included in works the least; used sparingly when included; and given more solo opportunities.

Although the names of the SPC groups are related to important predictions about their functions, the names are not intended to suggest that a group has only one gesture. The name simply refers to a salient gesture - each group might have multiple functions. The SPC model is a new and goal-oriented way to conceive of instrument combination: it is a broad division of instruments into three groups (Standard, Power, and Color), with each group having specific expressive characteristics. 


\section{TESTING THE SPC MODEL}

\section{Introduction}

The next stage of the study tests five predictions of the SPC model (using new samples for each hypothesis) to determine the strength and musical relevance of SPC group descriptions:

(1) Symphonic works include Color instruments less often than other instruments;

(2) If a Color instrument is part of the instrumentation, then the Color instrument tends to be used sparingly;

(3) Non-Color instruments do not solo with the same frequency as Color instruments;

(4) The deployment of Power instruments is positively associated with the loudness of dynamic markings; and

(5) If Power instruments are present in the musical texture, then the range utilized for the orchestra is wider than when Power instruments are absent.

The first three hypotheses explored the use of Color instruments: bass clarinet, contrabassoon, English horn, harp, and cornet. Since the sample used in the exploratory modeling of the present study was relatively small ( $n=50$ symphonic works) compared to the sheer number and variety of orchestral instruments, the Color group was limited to the above instruments. Although some of the pieces in the sample contained other rare instruments (e.g., serpent, ophecleide, Wagner tuba, tenor tuba, and bass trumpet), there were very few occurrences of these instruments - not enough to make inferences about their membership in a SPC group. Percussion instruments other than the timpani were also excluded from the study for the same reason. Relative rareness might be one trait of Color instruments, but it is not the sole determinant of Color function; it would be a mistake to include all rare instruments in the Color group at this point in time because they could function as extensions of other instrument groups.

\section{Hypothesis 1}

Symphonic works include Color instruments less often than other instruments.

\section{METHOD}

This hypothesis test used all Romantic-era symphonic works listed in Dubal (2001): $n=230$. Instrumentation lists in David Daniels' (2005) Orchestral Music: A Handbook was used to calculate the inclusion frequencies for each of the instruments investigated in the present study.

\section{RESULTS}

Figure 3 shows a frequency distribution for all 19 instruments; the average instrument had a probability of being included in the instrumentation $75.2 \%$ of the time (173 instances out of 230 total); the standard deviation of instrument inclusion frequency was equal to 74.6 instances. 


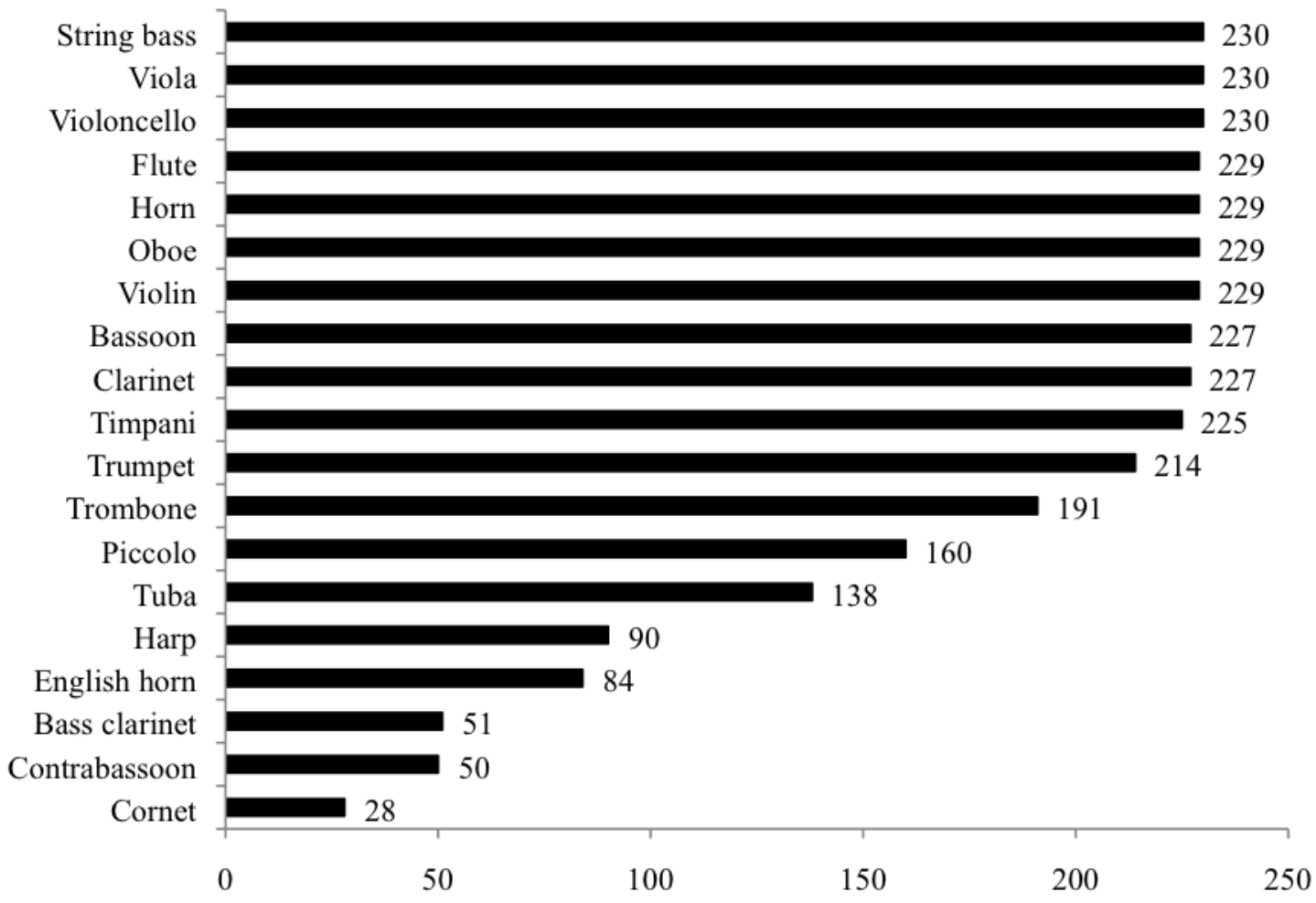

Figure 3. Number of inclusions of each instrument in 230 symphonic works; maximum $=230$, minimum $=$ $28 ; S D=74.6$ inclusions.

Table 2 compares each instrument's inclusion frequency relative to the average rate of inclusion. These $z$-scores show the standard deviation of each instrument's inclusion frequency relative to the mean. The distribution in Figure 3 suggests that many of the Color instruments lie at the low end of the inclusion continuum, but the z-scores in Table 2 make this more apparent - all Color instruments are more than one standard deviation below the mean inclusion frequency.

\begin{tabular}{ll} 
Instrument & $\boldsymbol{z}$-Score \\
\hline Viola & 0.76 \\
Violoncello & 0.76 \\
String bass & 0.76 \\
Violin & 0.75 \\
Horn & 0.75 \\
Flute & 0.75 \\
Oboe & 0.75 \\
Clarinet & 0.72 \\
Bassoon & 0.72 \\
Timpani & 0.70 \\
\hline
\end{tabular}

\begin{tabular}{ll} 
Instrument & $\boldsymbol{z}$-Score \\
\hline Trumpet & 0.55 \\
Trombone & 0.24 \\
Piccolo & -0.05 \\
Tuba & -0.47 \\
Harp & $\mathbf{- 1 . 1 1}$ \\
English horn & $\mathbf{- 1 . 1 9}$ \\
Bass clarinet & $\mathbf{- 1 . 6 4}$ \\
Contrabassoon & $\mathbf{- 1 . 6 5}$ \\
Cornet & $\mathbf{- 1 . 9 4}$ \\
\hline
\end{tabular}

Table 2. Instrument inclusion frequency relative to the average expressed as $z$-scores. Color instruments are shaded grey and their $z$-scores are shown in bold font. 
A formal test of the hypothesis examined the possibility of a significant difference between the inclusion frequencies of the Color group compared to average inclusion frequency. The null hypothesis assumed an equal probability of inclusion for all instruments in a symphonic work; the test hypothesis predicted that Color instruments are included less often than the average instrument. A one-tailed $t$-test compared the mean Color instrument inclusion frequency $(60.6$ inclusions; $S D=25.9)$ with the mean average instrument inclusion rate (173 inclusions; $S D=74.6)$ : the calculated $t$-score equaled -9.71 and was well beyond the critical $t$-value, which was $-1.53(\alpha=0.10$, one-tailed test). Thus, the null hypothesis that there is no difference between Color instruments and the average instrument was rejected. These results are consistent with the notion that Color instruments are included less often in symphonic works.

\section{CONCLUSION}

Although these results seem merely to support the obvious, the empirical confirmation of Color instruments' rarity is an important foundation for the SPC model. Infrequently used instruments become marked through their notable inclusions in certain pieces. However, the key point here is not so much the instruments themselves (although they might have some inherently contrasting qualities), but the effect of rarity. Tutti violin section performance is very common in symphonies, but the rest of the section does not double a concertmaster's violin solo - this context for violin takes on a quality of contrast by virtue of being an infrequent occurrence. Thus, one can take the fact that Color instruments lie more than one standard deviation below the average instrument inclusion frequency, and generalize the overall Color gesture to other instruments.

\section{Hypothesis 2}

If a Color instrument is part of the instrumentation, then the Color instrument tends to be used sparingly.

Hypothesis 1 naturally suggests this follow-up prediction based upon the idea of limited scoring of Color instruments: when Color instruments are included in a piece, they are peppered throughout the work and held on reserve for special moments. Minimal, yet noticeable, performance time would be a characteristic of a musical function that acts to interrupt, divert, and offset the main tone colors of a piece of music.

\section{METHOD}

Hypothesis 1 revealed that the average instrument is included in the instrumentation $75.2 \%$ of the time. The current hypothesis calculated the probability that the average instrument actually performs when it is included in a piece. The average instrument's probability of performance was calculated by randomly sampling 300 measures: twenty measures from fifteen different works. In each of the sampled measures, the number of instrument timbres was counted (totaling 2513). In addition, the total number of opportunities available for an average instrument timbre to perform was calculated for the piece (totaling 5060).[3]

All of the information taken from each piece was collapsed into a single calculation. Table 3 summarizes the calculation. In the 300 measures, there were 5060 opportunities for an instrument to perform. Of those opportunities, 2513 instruments were actually deployed. Therefore, the average instrument has a probability of playing $49.66 \%$ of the time in a symphonic work from the Romantic era.

\begin{tabular}{lll}
$\begin{array}{l}\text { Number of } \\
\text { instruments } \\
\text { present }\end{array}$ & $\begin{array}{l}\text { Total number of } \\
\text { opportunities to perform }\end{array}$ & $\begin{array}{l}\text { Average instrument } \\
\text { performance frequency }\end{array}$ \\
\hline & & \\
2513 & 5060 & $49.66 \%$ \\
\hline
\end{tabular}

Table 3. Derivation of the performance probability for an abstract average instrument. 
Next, the investigation turned to individual Color instruments. For each instrument, eight pieces were randomly sampled from the total number of pieces that include the specific Color instrument. Within each piece, fifteen bars (non-contiguous) were randomly sampled. Then, the presence or absence of the Color instrument was recorded for each bar. The total number of bars that the Color instrument plays was then used to calculate a probability that the Color instrument would play in the piece. This process was repeated for all eight pieces and then the average probability was calculated for each instrument.

\section{RESULTS}

Table 4 shows the average performance probabilities for each Color instrument. In addition, the table shows $t$-scores for each instrument relative to the average instrument's inclusion (7.46 performing moments/15 opportunities).[4] One-tailed $t$-tests were conducted for all Color instruments $(\alpha=0.10)$. The null hypothesis assumed no difference between the frequency of performance of Color instruments and the average instrument. The hypothesis proposed that Color instruments perform significantly less than the average. The critical $t$-score was equal to -1.415 .

\begin{tabular}{llll}
\hline Instrument & $\begin{array}{l}\text { Performance frequency average } \\
\text { (out of 15 total opportunities) }\end{array}$ & Percentage & Sample size \\
\hline & number of occurrences & $\%$ & $n$ \\
Bass clarinet & 6.63 & 44.17 & 8 \\
Contrabassoon & 4.88 & 32.53 & 8 \\
Cornet & 4.75 & 31.67 & 8 \\
English horn & 4.00 & 26.67 & 8 \\
Harp & 2.71 & 18.10 & 7 \\
& & & \\
\hline Instrument & Standard deviation & $\boldsymbol{t}$-Score \\
\hline & $S D$ & $t$ & \\
Bass clarinet & 2.39 & -0.98 \\
Contrabassoon & 2.30 & -3.17 \\
Cornet & 3.54 & -2.17 \\
English horn & 3.38 & -2.90 & \\
Harp & 3.63 & -3.46 & \\
\hline
\end{tabular}

Table 4. Performance frequencies and $t$-scores of Color instruments from samples of eight different works per instrument.

The one-tailed $t$-test results show that nearly all of the Color instruments' performance rates are located more than two standard deviations away from the mean. The only exception is the bass clarinet. Therefore, in the case of the harp, English horn, cornet, and contrabassoon, the null hypothesis (that Color instruments play the same amount as other instruments) was rejected. The results are consistent with the notion that when Color instruments are included in a work's instrumentation, they perform significantly less than the average instrument.

\section{CONCLUSION}

Through less performance time, Color instruments would gain an advantage of low listener habituation: if "auditory fatigue" is related to habituation and attention span, then Color instruments could serve to refresh the orchestral texture and maintain listener engagement with the music. In the case of the bass clarinet, the null hypothesis failed to be rejected. The mean frequency of bass clarinet performance was not significantly lower than the mean performance frequency of the average instrument, but it was skewed in the predicted direction of infrequent use. Taken alone, this result might suggest that the bass clarinet is not a Color instrument, but a member of another SPC group; alternatively, it could have some Color attributes combined with some traits from other SPC model groups. 


\section{Hypothesis 3}

Non-Color instruments do not solo with the same frequency as Color instruments.

This hypothesis tested one important prediction of Color function: if a Color instrument's sparing use is indeed intended to give it an ear-catching contrasting quality, then a Color instrument's appearance would align with solo moments in music more so than other instruments. On one hand, composers might prefer to give solos to Color instruments rather than other instruments because Color instruments' generally sparing use lends them an automatic timbral contrast; and their softer tones are best heard in contexts that minimize masking. On the other hand, solos might be a fairly common occurrence-composers potentially prefer timbral variety rather than timbral contrast.

\section{METHOD}

Defining a "solo" proved to be the most challenging element of this hypothesis test. The spectrum of sololike moments includes many types of musical configurations; for example:

- A lead voice within a texture of multiple duplications of a melodic line

- A unique and melodically prominent, yet accompanied line

- An instrument that plays completely alone

The present study hoped to identify solos that are a combination of the second and third bullet points above, but it was difficult to identify solos using an objective decision process. Due to this difficulty, solo moments were identified via the expertise of four other musicians who had no knowledge of the hypothesis currently under investigation. At the time of this study they were graduate students in music theory, composition, and conducting at Ohio State University. Each musician was given a detailed instruction sheet that included a definition of a solo: "One might argue that it is possible to perceive multiple, simultaneous solo lines, but the present study will exclude this option and consider a true solo as a line of primary importance that is used in only a single instrument color. The solo will be defined as a line that does not have to compete with any simultaneous, rival lines. The solo usually has support from lines of significantly less prominence; sometimes it occurs completely alone." Seventy randomly-selected pages from the sampled works were given to each musician. Then, the participants used a marker to highlight the soloing instrument for the complete duration of its solo.

Once the identified solos were collected from the musicians, each highlighted solo passage was matched with a randomly-selected measure from the same page that contained no solos. This step preserved the independence of each observed solo, while balancing the moment with a non-solo passage with the same potential for instrumentation.

The pairing technique defined a "solo reserve coefficient." This metric is a scale ranging from 0.5 to 1.0 - a hypothetical instrument used only for solo moments would have, for example, 10 solo moments and 0 non-solo moments. The total number of solo measures divided by the total number of measures performed would be $10 / 10=1.0$. The converse situation would be an instrument that played in non-solo sonorities just as often as it soloed. In this case, there might be 20 solo measure and 20 appearances in nonsolo measures. The ratio of solo measures to the total number of appearances would be $20 / 40=0.5$. 


\section{RESULTS}

Table 4 shows the frequency of solo and non-solo moments appearances for the Color and non-Color instruments that had solos in the randomly selected sample of 280 pages extracted from 70 different works.

\begin{tabular}{|c|c|c|c|c|}
\hline Instrument & $\underline{\text { Solos }}$ & $\underline{\text { Non-solos }}$ & Measures present & $\underline{\text { Solo reserve }}$ \\
\hline Bassoon & 3 & 3 & 6 & 0.5 \\
\hline Violin & 34 & 22 & 56 & 0.61 \\
\hline Horn & 12 & 7 & 19 & 0.63 \\
\hline Oboe & 14 & 7 & 21 & 0.67 \\
\hline Trumpet & 4 & 2 & 6 & 0.67 \\
\hline Violoncello & 10 & 5 & 15 & 0.67 \\
\hline Viola & 10 & 5 & 15 & 0.67 \\
\hline Harp & 2 & 1 & 3 & 0.67 \\
\hline Clarinet & 8 & 4 & 12 & 0.67 \\
\hline Flute & 12 & 5 & 17 & 0.71 \\
\hline English horn & 2 & 0 & 2 & 1 \\
\hline Trombone & 1 & 0 & 1 & 1 \\
\hline Triangle & 1 & 0 & 1 & 1 \\
\hline String bass & 1 & 0 & 1 & 1 \\
\hline
\end{tabular}

Table 4. Descriptive statistics of the experts' solo identifications and the randomly sampled non-solo measure. This table is not statistically reliable because the number of sampled measures varied widely between instruments; several instruments had a very small number and this posed additional difficulties.

Since the solo data in this hypothesis test were limited in number, it was not possible to perform any inferential statistics between Color instruments and all other instruments at this stage of sampling.

\section{CONCLUSION}

Perhaps the most insightful aspect of the current hypothesis was not the frequency of Color instrument solos, but the frequency of a solo in general. True solos are not as common as one might predict. Another interesting aspect of the results is that many instruments seem to move towards a solo reserve coefficient of 0.67. This number could be an artifact of the small sample, but it suggests a nineteenth-century practice of balancing solo moments with half the number of non-solo moments. Thus, for those instruments that solo in a particular work, there might be a tendency for composers to avoid the overuse of those soloists in the vicinity of their solos.

\section{Hypothesis 4}

The deployment of Power instruments is positively associated with the loudness of dynamic markings

In the MDS map of instruments (Figure 1), the dispersion of instruments along the horizontal axis (Dimension 1) of the figure suggests that dynamic potential is an important determinant of instrument function in the orchestra. The contrast between instruments at the left and right ends of the graph is consistent with musical intuitions of subdued (on the left) versus more powerful (right) instruments. If composers used these instruments to convey an auditory impression of power, then one might expect an explicit link of Power instruments with loud dynamics. 


\section{METHOD}

An ordinal scale was assigned to eight common dynamic markings. The scale shown in Table 5 represents the loudness hierarchy of dynamic markings and preserves the variance of dynamic markings without dichotomizing dynamic levels into only two categories, loud and soft.

$\begin{array}{lcccccccc}\text { Dynamic: } & p p p & p p & p & m p & m f & f & f f & f f f \\ \text { Loudness Rank: } & 1 & 2 & 3 & 4 & 5 & 6 & 7 & 8\end{array}$

Table 5. Ordinal scale of dynamic strength ratings.

Forty works containing all SPC functional category types were sampled. Within each piece, every dynamic marking was considered a valid sampling point, but only if there was no dynamic marking discrepancy between instruments. For example, if the oboe was marked $f f$ and the clarinet was marked $m f$, then that point in the music was not included. Each sampling point was a complete measure containing equal dynamic markings in all instruments. Any marking other than those in the ordinal scale were not used in the sample.

The dynamic strength and number of Power instruments playing in the sampled measure was recorded. For example, sampled measure $\mathrm{x}$ was selected because there was a consensus between all instruments on a dynamic marking of $m f$. This marking was coded as " 5 " and then the presence of the trombone, timpani, and trumpet was recorded as " 3 " Power instruments. Therefore, sampled measure $\mathrm{x}$ was represented by the number pair " $5-3$. "

\section{RESULTS}

A Spearman rank correlation coefficient established the relationship between the ordinal rank of dynamic level and the number of Power instruments present in the sampled sonorities. In a total of 1162 measures the correlation between numbers of Power instruments and dynamic strength was $r_{\mathrm{s}}=0.504(p<0.01)$. This moderate, positive correlation suggests that there is a relationship between dynamic strength and the number of Power instruments present in a texture. The results are consistent with the hypothesis that the presence of a Power instrument is associated positively with dynamic loudness levels.

\section{CONCLUSION}

Although Power instruments' order of entry during increases in dynamic strength was not specifically tested in this hypothesis, it was observed that the horn is nearly ubiquitous at all dynamic levels. During the score scanning process, it was evident that if only a single Power instrument was present in a texture, then it was most likely to be the horn. It was usually the first Power instrument added during a large-scale crescendo and it was commonly the last Power instrument to be removed from the orchestration during a decrescendo. Nevertheless, the Power group as a whole is associated with louder dynamics.

\section{Hypothesis 5}

If Power instruments are present in the musical texture, then the range utilized for the orchestra is wider than when Power instruments are absent:

- Treble instruments will have higher pitch height when Power instruments are used OR

- Bass instruments will have lower average pitch height when Power instruments are used

The results of the previous hypothesis test supported the idea that Power instruments tend to play at louder dynamic levels. In addition, Power instruments possibly influence the orchestra's use of pitch extremes. It 
seems counter-intuitive to include the piccolo and tuba into the same functional group; however, the Power instruments share not only loud dynamics, but also extreme register (both high and low pitch heights). Although it would be most compelling if Power instruments pushed the pitch range outward in both the high and low directions, it is possible that Power instruments might expand overall pitch range in one direction: down or up. Hence, two sub-hypotheses (bulleted under the general hypothesis statement) were tested to understand the precise direction of pitch range expansion.

\section{METHOD}

The initial method, considered for this hypothesis test, planned to 1) sample random sonorities from scores; 2) calculate the pitch height for all performing treble and bass instruments; and 3) average all instruments' pitch heights together to produce an average treble and bass pitch height for every sonority. While this approach would have precision, it lacks control over the number of instruments playing in the sampled sonorities: the sheer number of instruments (regardless of type) could influence the calculated average pitch range. The following sampling method was used to eliminate the potential confound caused by different numbers of instruments playing at any given point in time of a symphonic work.

The range fluctuations of two instruments (violin and violoncello) were used as the gauge from which inferences about the composite range of the orchestra could be made. These instruments were chosen because of their Standard classification: it was likely that they would perform frequently. The violin represented the treble range instruments, while the violoncello represented the bass range instruments. If the addition of Power instruments into the texture has an association with wider pitch range, then one would expect to see a rise in the violin's average pitch height, or a falling of the violoncello's average pitch height.

The present study collected both of its samples using two different criteria: 1) no Power instruments present ("non-Power" sonorities) and 2) one or more Power instruments present ("Power" sonorities). The non-Power sample comprised up to three sonorities randomly sampled from 32 randomlyselected works ( $n=84$ sonorities).[5] Each page was scanned from beginning to end, stopping at the first sonority that contained violin, violoncello, and no Power instrument. If nothing on the page met these criteria, then successive pages were searched in turn until a non-Power sonority was found. Steps were taken to prevent accidental re-sampling of any previously sampled sonority. The semitone distances between each sampled pitch and D4 were averaged together to determine the mean pitch height (Table 6) for the violin and violoncello in non-Power contexts.[6] The second comparison distribution contained only sonorities using one or more Power instruments and the violin and violoncello. The sampling technique and mean pitch-height calculation (Table 6) mirrored the non-Power stage outlined above.

\section{Non-Power Sonorities}

\begin{tabular}{llll} 
& Mean (semits) & Standard Deviation (semits) & $n$ \\
\hline Violin height & 11.50 & 8.60 & 84 \\
Violoncello height & -10.79 & 7.58 & 84 \\
\hline
\end{tabular}

\section{Power}

\begin{tabular}{llll} 
& Mean (semits) & Standard Deviation (semits) & $n$ \\
\hline Violin height & 13.02 & 9.21 & 86 \\
Violoncello height & -10.52 & 8.70 & 86 \\
\hline
\end{tabular}

Table 6. Descriptive statistics for average pitch height, in relation to D4, of violin and violoncello in nonPower and Power sonorities. 


\section{RESULTS}

The hypothesis test used two $t$-tests (one-tailed) to compare fluctuations of average pitch height in Power and non-Power sonorities:

The treble range sub-hypothesis predicted that the sonorities containing Power instruments would have a higher average pitch those without Power instruments. The first $t$-test compared the range of the violin between the Power and non-Power samples. The critical $t$ value using an alpha level of 0.10 was $t_{\text {crit }}$ $=1.292(d f=80)$. The calculated $t$ value was $t=1.11$, which was smaller than the critical $t$ value-there was no significant difference of the violin's average pitch height in non-Power sonorities versus Power sonorities.

The bass range hypothesis predicted that Power instruments contribute to a downward push of pitch-height in the orchestra's bass instruments - represented by the violoncello. Again, the critical $t$ value was equal to 1.292 . The calculated $t$ score for the violoncello was $t=0.210$. This also did not meet the criteria for statistical significance: the presence of Power instruments does not seem to lead to a significant lowering of violoncello pitch height.

\section{CONCLUSION}

Since there was not a statistically significant shift of violoncello or violin pitch height between non-Power and Power sonorities, there was no support for the overall hypothesis that the presence of Power instruments is associated with a widening of the average orchestral pitch range. The data collection process and score study informally reinforced that there is no discernible change of pitch height in Power compared to non-Power sonorities. Of course, this all rests upon the assumption that the violin and violoncello are representative of other instruments' pitch height. A future test of this hypothesis should collect pitch-height data from all orchestral instruments; it also should make a distinction between the number of Power instruments present in a texture. The "one or more" sampling criterion was convenient, but could be imprecise if the number of Power instruments significantly affects pitch range expansion. Power instruments are indeed dynamically forceful (Hypothesis 4), but they do not seem to function as both power and range expansion agents.

\section{General Conclusion}

Formal tests supported three out of five hypotheses based upon the SPC model; these are mixed results, but the moderate robustness of the SPC model warrants further investigation. Considering that these five hypotheses are a subset of a larger number of predictions that could be made by the SPC model, it is appropriate to reserve judgment about the degree of the model's accuracy until future studies are conducted.

Color instruments had a lower likelihood of inclusion and performance in orchestral works - they are held on reserve. Although they do not appear to have more solos than other instruments, Color instruments still could stand out from the rest of the orchestra if they have an inherently distinctive timbre, capable of projecting through, under, or above other groups of instruments.

Power instruments exhibited their namesake characteristic: dynamic strength and numbers of Power instruments present in the musical texture were moderately correlated. The intuition that Power instruments also function to expand the range of the orchestra did not gain empirical support. Power instruments seem to have little effect on the pitch height of other instruments; perhaps Power instruments themselves are the pitch register "icing on the cake" and solely contribute to a wider orchestral pitch range - this question was not tested in hypothesis 5 and could be tested in a future study. Hypothesis 5 also assumed that the violin and violoncello were adequate representatives of the orchestra: this might be false, so judgment should be suspended until the pitch range test is repeated with more precise measures of the whole orchestra's pitch range.

No hypotheses were tested for the Standard instruments. A few predictions were formulated, but none were deemed important given the restricted scope of the present, exploratory study. 


\section{DISCUSSION}

Although the present study puts forward a model of instrument combination in orchestration, this approach is descriptive and should not be interpreted as a prescription of orchestration patterns. Nor should the study of orchestration in the Romantic period be construed as a radical effort to reinstate "ideal" principles of composition. Rather, this approach intends to open up new insights into existing orchestration practices and possibly to inspire the creation of original, artistic ideas. The terms "Standard," "Power," and "Color" were chosen from several alternatives because SPC best encapsulates some important musical gestures in the nineteenth-century style of orchestration.

\section{SPC NAME ALTERNATIVES}

Examples of alternative names for the SPC groups: 1) Patterns of instruments in groups could be seen to reflect a historical progression of instrument use in symphonic music. One might use terms like "Old", "Newer", and "Newest" (ONN) - these adjectives refer mainly to the time of instruments' adoption into the orchestra and not their absolute age. The Standard instruments (plus the trumpet, horn, and timpani) comprise the complete orchestra used in the Classical period. The Power group contains instruments like the trombone, which existed long before the nineteenth century (i.e., the sackbut), but were newer additions to the symphonic genre and gained more freedom of expression via major technological advances in the Romantic period (e.g., valves for trumpets and horns). The Color group has instruments that either did not exist until the nineteenth century (e.g., the cornet) or were significantly improved in the nineteenth century to warrant their use in the orchestra. The ONN group names were not used because they only loosely reflect instrument timelines; plus, it is unlikely that composers would distribute instruments solely based upon their "age" in the orchestra. 2) Alternatively, SPC groups might be called "Most Frequent," "Less Frequent," and "Least Frequent" (MLL) in reference to instrumentation frequency; Figure 3 suggests this stratification - with exception to the horn - but the MLL explanation is weaker than the SPC model. MLL does not suggest a goal-oriented element in instrument use - for example, that Power instruments are well suited to loud dynamics or that Color instruments are lower or warmer variants of other instruments.

\section{LIMITATIONS AND PROMISES OF THE SPC MODEL}

By no means does the SPC motto preclude other large-scale functions or confine instruments into a single category; rather, the SPC model suggests that composers sometimes combine entire instrument families (Standard $=$ Woodwinds + Strings), or combine instruments between instrument families. The resulting combinations look to be aligned with three purposes: 1) Establish a tone-color foundation and reference point (Standard); 2) Provide loud dynamic contrasts and climaxes (Power); and 3) Create timbral contrast using rarer, lower, or more mellow instruments (Color). It would be very exciting to see other functions emerge from further study of both the nineteenth century and other time periods; an important place to start is with percussion instruments, which were excluded from the SPC model.

The present study used only snapshots in time as representatives for orchestration patternspatterns that are, in reality, continuously in flux during performances of symphonic works. Future studies could look at temporal variables such as instrument entrances and exits from the texture during crescendi and decrescendi, for example. Additionally, it would be important to show that orchestral gestures are primary and the instruments in those groups are secondary: if this is true, then one might expect to see a stable repertoire of gestures, but with instruments that can assume alternative roles as they dissolve away from one group and join another group. Instrument fluidity in conjunction with gestural stability seems to be one characteristic of twentieth-century orchestration, which combines instruments quite flexibly and imaginatively; instrument combinations appear to fulfill a number of roles depending upon the use of extended techniques (e.g., multiphonics; altissimo effects; key slaps) or the use of mutes.

The SPC model has broad applications for the development of new musical analysis techniques and also for the continued construction of orchestration theory; there are fruitful insights that lie ahead. Despite orchestration's vast importance, it has eluded many musical analysis techniques. As a consequence, music-theoretic approaches generally have not treated concerns of orchestration in as great of depth as other primary musical parameters (e.g., harmony, melody). This is not surprising considering western art music's notation, which explicitly specifies pitch and rhythm, but imprecisely represents other vital sound characteristics like tone quality and balance (Palmer, 1997). Orchestration treatises largely discuss the first- 
order properties of individual instruments, but these issues are more appropriately thought of as "instrumentation" issues. Orchestration involves the higher-order processes of instrument combination, instrument orderings, pitch distribution among instruments, and many other elements — ones that could lead to bountiful musical discoveries if future studies examine them in detail. The SPC theory not only describes the impetus of gesture on instrument combination, but the method also shows how a hybrid artisticempirical approach can take the multi-faceted contributions from diverse fields of inquiry and bridge them with orchestration theory.

\section{NOTES}

[1] Typical chapter subheadings are organized around instrument families. For example: "Scoring for Strings" and "Scoring for Woodwinds," see Blatter (1997) and other orchestration texts (Piston, 1955; Rimsky-Korsakov, 1964[1913]). While headings concerning combinations across instrument families do occur, the treatment of this topic is minimal when compared to the treatment of scoring within families.

[2] The Proxcal 1.0 algorithm by the Data Theory Scaling System Group (DTSS) of the Faculty of Social and Behavioral Sciences, Leiden University, The Netherlands, was used for this analysis. The present study used 10 random starts for the initial configuration of the instruments; interval transformation was used for the dissimilarity measures.

[3] Percussion instruments were included in this calculation. The percussion section might operate as a single entity in some cases, but at the same time, the section is composed of many different timbres. The calculation considered each different percussion instrument as a separate timbre opportunity.

[4] The value of 7.46 came from the average instrument's performance frequency $(49.66 \%)$ relative to 15 performance opportunities.

[5] The non-Power sonority sample was slightly smaller than the expected $n=96$ because it was overly time-consuming to search for non-Power sonorities that had a nearly constant presence of one or more Power instruments: sometimes pieces appeared to have less than three instances of a non-Power sonority. These examples were usually shorter symphonic pieces, but this observation is nevertheless a challenge to the notion that Power instruments provide contrast to the prevailing orchestration - Power instruments are sometimes quite prevalent.

[6] D4 represents the average notated pitch of western classical music; see Huron (2001).

\section{REFERENCES}

Blatter, A. (1997). Instrumentation and Orchestration (2nd ed.). New York: Schirmer Books.

Caclin, A., McAdams, S., Smith, B.K., \& Winsberg, S. (2005). Acoustic correlates of timbre space dimensions: A confirmatory study using synthetic tones. Journal of the Acoustic Society of America, Vol. 118 , No. 1, pp. 471-482.

Daniels, D. (2005). Orchestral Music: A Handbook (4th ed.). Lanham, MD: Scarecrow Press.

Dubal, D. (2001). The Essential Canon of Classical Music. New York: North Point Press.

Grey, J. (1977). Multidimensional perceptual scaling of musical timbres. Journal of the Acoustical Society of America, Vol. 61, No. 5, pp. 1270-1277.

Huron, D. (2001). Tone and voice: A derivation of the rules of voice-leading from perceptual principles. Music Perception, Vol. 19, No. 1, pp. 1-64. 
McAdams, S., Winsberg, S., Donnadieu, S., De Soete, G., \& Krimphoff, J. (1995). Perceptual scaling of synthesized musical timbres: Common dimensions, specification and latent subject classes. Psychological Research, Vol. 58, pp. 177-192.

Palmer, C. (1997). Music performance. Annual Review of Psychology, Vol. 48, pp. 115-138.

Piston, W. (1955). Orchestration. New York: Norton.

Rimsky-Korsakov, N. (1964). Principles of Orchestration (M. Steinberg, Ed. \& E. Agate, Trans.). New York: Dover. (Original work published 1913).

Sandell, G.J. (1991). Concurrent Timbres in Orchestration: A Perceptual Study of Factors Determining "Blend". Ph.D. dissertation. Northwestern University. 